\title{
Dynamic valuation function based definition of the primary spectrum user in collocated cellular networks
}

\author{
Michal Szydelko, Jaroslaw Byrka, Jakub Oszmianski \\ Wroclaw Research Center EIT+, Stablowicka 147, 54-066 Wroclaw, Poland \\ \{Michal.Szydelko, Jaroslaw.Byrka, Jakub.Oszmianski\}@eitplus.pl
}

\begin{abstract}
We propose to use generalization of a second price VCG auction to dynamically allocate shared radio resources among collocated, equally prioritized mobile network operators in multi carrier HSDPA cellular network. The spectrum usage priority will be dynamically decided based on the comparison of individual valuation functions, used as an input for the spectrum auctioning game. After theoretical introduction to the game theory, we demonstrate that short term auction based spectrum sharing provides spectrum utilization gains for coalition of MNO's having un-equall cell specific traffic loads, allowing network operators to secure their business and at the same time, open new market possibilities with additional revenue opportunities.
\end{abstract}

Keywords: Auctions, valuation function, spectrum broker, spectrum trading, spectrum sharing, primary user, game theory, VCG, HSDPA

\section{INTRODUCTION}

Spectrum resources sharing, as well as its flexible realization of dynamic spectrum access, has become very popular research topic, not only due to its research potential, but also due to the increasing market interest in larger revenues for Mobile Network Operators (MNO), as well as from regulatory and policy makers point of view, where the main interest is in increasing end-user satisfaction and in improving the spectrum utilization [1], [2], [3]. With the rapid growth in the number of mobile terminals, constantly increasing demands for the mobile services generated data rates, the limitation of spectrum allocation is becoming bottleneck for many cellular network operators. Therefore, physical radio resources sharing has been widely considered for the future of wireless communications, both to overcome the scarcity of spectrum as well as to decrease Operational Expenses (OPEX) of cellular networks.

Spectrum sharing permits the network operators to tune their radio resources allocation dynamically, according to varying traffic demand. In addition, sharing network elements reduces the cost of acquisition and maintenance of the network per operator [4], [6]. Based on these principles, we are making an attempt to model cellular network radio access, considering conditional and opportunistic access to the shared radio resources, where the condition is formulated based on the game theory driven principles of valuation functions. This approach is seen as one of solutions, to avoid channel sensing complications of the cognitive radio and an attempt to allow more flexible spectrum usage in cellular networks, allowing dynamic declaration of the primary and secondary spectrum usage based on the subscribers demands.

In this context, different resource sharing solutions, utilizing auctioning concepts have been proposed in the literature. In [17] two auction mechanisms for allocating the received power were proposed. The first was an auction in which users were charged for the received SINR, which, when combined with logarithmic utilities, lead to a weighted max-min fair SINR allocation. The second was an auction in which users were charged for power, which maximized the total utility when the bandwidth was large enough and the receivers were colocated. Both cases were motivated by the scenario in which users wish to purchase a local, short-term data service. In our approach we provide a solution, which requires much less information exchange between auction players, than the concepts mentioned above. We propose to use Vikrey, Clarke and Groves (VCG) mechanism (generalized from the second price auction concept) for equal value, multi-unit auctions in the context of radio resource allocation for cellular networks. VCG mechanism is a standard concept in Algorithmic Game Theory and its main advantage is the combination of two properties: maximization of social value and incentive compatibility. Incentive compatibility ensures that participants do not gain by bidding different than their true valuations, and hence we will refer to bids as valuations of resources submitted to the auctioneer. Our auction-based mechanism can be seen as a compromise between two approaches:

- to jointly schedule the total traffic from all service providers involved in auctions (maximizing radio resources utilization), or

- to have orthogonal radio resources dedicated to each operator for his exclusive usage (no information exchange required).

For that, we introduce a third party virtual Auctioning Unit (AU), responsible for regulating the usage/pricing of the radio resources based on received operators valuations.

The paper is organized as follows. First we describe standard features of the VCG mechanisms which were used as the engine for the spectrum resources distribution, in Section II. Section III is dedicated to the utility functions, which were 
used as the decision criteria for the primary user definition, among participating, concurrent parties. Finally, system level simulation results are presented in Section VI, based on the model described in Section IV and V.

\section{VCG MECHANISMS}

Vikrey, Clarke and Groves, proposed in a series of papers a class of mechanisms for distributing goods to users. The common feature of these mechanisms is that they produce socially optimal distributions, at the same time remaining incentive compatible [12], [13], [14], [8].

Intuitively, the way to obtain incentive compatibility is to charge each user $i$ the amount by which the other users suffer from $i$ being in the system. In this construction, the payment of $i$ does not directly depend on his submitted valuation (for the goods to be distributed), but on the valuations of other users and the distribution itself.

Consider a game played by $m$ players interested in $n$ identical goods. Each single player $i$ has his utility from obtaining a certain amount of these goods. Let $[n]$ be a shorthand for $\{1,2, \ldots, n\}$. Each player submits a function $V_{i}:[n] \rightarrow R$ called valuation to the auctioneer, which assigns goods to players based on their valuations and computes payments. We will assume the utilities and therefore also the submitted functions are submodular, that is the profit from having one more good decreases with the number of goods already obtained.

The main objective of the auctioneer is to maximize the utilization of the goods (social value maximization) and therefore he attempts to assign goods so that the total utility is maximized. The following simple VCG mechanism is known for having this property.

For each player $p_{i} \in P$ define $P^{-p_{i}}=P \backslash\left\{p_{i}\right\}$ to be the set of all players except $p_{i}$.

For any subset of players $S \subset P$, we define an assignment of $n$ goods to $S$ to be a function $f: S->[n]$ such that $\sum_{p \in S} f(p) \leq n$, i.e., such that the total number of assigned goods is at most the number of available goods $n$. Given an assignment $f$ we define its social value to be the total value the players get from the goods assigned to them in the assignment $f$. More formally, the social value of an assignment $f$ is denoted by $\operatorname{Val}(f, S)=\sum_{o_{i} \in S} U_{i}\left(f\left(o_{i}\right)\right)$, i.e. the total utility of players given assignment $f$.

Observe, that given the utilities of the players it is trivial to calculate $\operatorname{Val}(f, S)$, moreover given the utility functions one may find the social value optimizing allocation by running a simple greedy algorithm. This algorithm looks at the marginal profits of obtaining one more good, and considering goods one by one, allocates the next good to the player whose marginal utility is the highest. The crux of the VCG method lies in revealing the utilities to the auctioneer. One argues that if the prices charged to the players are carefully chosen, players will have no incentive in submitting bids, different then their valuations. Intuitively the users have to pay for the damage they make to the others. We will now specify the payments in more details.
Consider the total profit a group of players $S \subset P$ may make from using $n$ goods. We call this maximal total profit the $n$-goods value of the set $S$, and we denote it by $\operatorname{Val}_{n}(S)=$

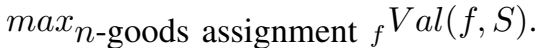

The mechanism is defined as follows:

- Distribute goods according to an assignment $f^{*}$ of $n$ goods that maximizes $\operatorname{Val}(f, P)$,

- Charge each player $p_{i}$ the amount of money equal $\operatorname{Val}_{n}\left(P^{-p_{i}}\right)-V a l_{n-f^{*}\left(p_{i}\right)}\left(P^{-p_{i}}\right)$, i.e. the amount by which the total value for the other players gets worse because of $p_{i}$ using his $f^{*}\left(p_{i}\right)$ goods.

The charge may simply be computed by repeatedly applying the greedy algorithm to the setting with one player removed. Such computation would require $2 m$ runs of the greedy procedure and would then require roughly quadratic time. Note however, that one may compute the valuations all together by considering a sorted sequence of merged marginal valuations of the different players.

Assuming the submitted valuations of the players are truthful, the computed assignment of goods is by definition the one that maximizes the total profit of the players. It remains to argue that no player has any incentive to declare valuation different from his true valuation, associated with his utility. This property of the mechanism called incentive compatibility is the focus of game theory. It is known that VCG mechanism are essentially the only possibility for truthful auctions [8].

Application of a VCG mechanism is only possible if the underlying (social value) optimization problem is computationally easy. We may obtain this by assuming that the distributed goods are of the same value and that the players' valuation functions are submodular. Not only it is an acceptable restriction from the application perspective, it is also vital for the construction of the mechanism.

By allowing valuations that are not submodular the optimization problem becomes NP-hard, which may be shown by a reduction from the Knapsack problem. For auctions with heterogeneous goods, still assuming submodular valuations, the joint utility optimization can only be approximated with $(1+1 / e)$ factor [9] without the incentive compatibility property, and with a logarithmic factor by an incentive compatible mechanism [10]. In case of heterogeneous goods and nonsubmodular valuations the problem becomes $\sqrt{n}$ hard to approximate [11] even without incentive compatibility.

\section{ApPlicAtion to SPECTRUM Distribution AND UTILITY FUNCTIONS}

The main requirement for the players to participate in the described mechanism is to estimate their demand for goods and to quantify this demand in terms of money they are willing to pay for each granted good's unit. As we believe, only the player himself can estimate the utility of goods, his utility is not directly known to the mechanism. The only information the mechanism has is the valuation reported by the player. The calculation of utility functions may consider different factors and it's completely subjected to the players' judgment. For the operators to participate in the auction they have to decide, 
depending on their policies for traffic and user prioritization, QoS and environmental conditions, how important it is for them to succeed in transferring a certain portion of data.

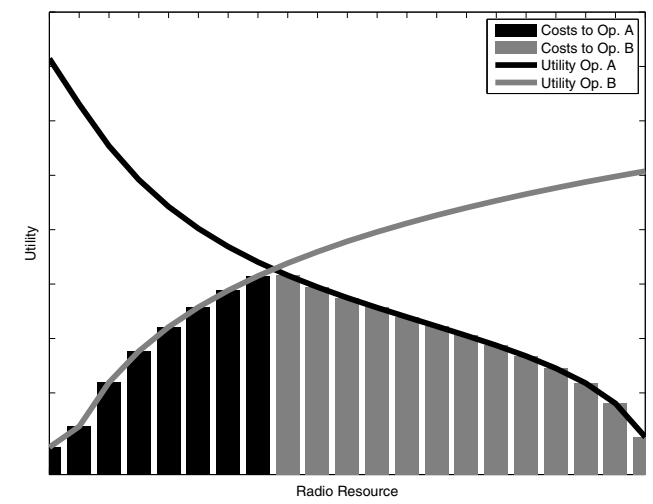

Figure 1. Example realization of cell specific resource distribution based on the valuation functions for 2 operators. The split of resources is on the intersection of the valuation curves.

We believe that a fairly accurate estimation can be computed for short time horizons (within range of seconds) where the number of active connections is not expected to vary too much and the traffic profiles can be recognized.

\section{A. Realization of example valuation}

One of the determining factors in the calculation of the valuation function is the amount of data to be transmitted in the next period of time $\Delta$. Therefore, an easy way of determining the valuation would be a ratio between the amount of buffered data and the number of resources. Assuming $Q_{k}$ to be the pending cell specific data to be transmitted by operator $k$ its valuation for getting $x$ resources could be given by:

$$
U_{k}(x)=\alpha \sum_{i=1}^{x} \frac{Q_{k}}{i}=\alpha Q_{k} \cdot H_{x} \approx \alpha Q_{k} \cdot \ln (x),
$$

where $\alpha$ represents a scaling constant to convert the values into realistic price, and $H_{i}$ is the $i$-th harmonic number. NOTE: This function is closely related to proportional fairness scheduling rules.

If all players calculate their valuations this way, the resources will be distributed proportionally to the current $Q_{k}$ values. Moreover, each operator $o_{k}$ has the guaranty that the total paid fee will be at most $\alpha Q_{k}$, even if the other players decide to use different valuation methodologies.

\section{Analytical Model}

Consider two network operators who are interested in providing mobile services, using a particular Radio Access Technology (RAT), over the same geographical area which is typical in case of highly populated locations. We assume these operators have collocated Base Stations (BS), which is a reasonable assumption since this approach is already widely applied in the market for the network cost reductions[7]. Such model is not typical cognitive radio model, as both network operators are considered as primary users at the initial phase - we rather investigate, whether each of them is utilizing its own spectrum resources in the optimal way. Furthermore, this does not mean that the modeled concept is to punish the less loaded or under-utilized network operator, as the described carriers auctioning is, or can be, based on the money flows being a compensation for the potentially non-served load in the donor's network.

The described spectrum auctions do not introduce any limitations to the players set. However, from the practical consideration as well as for the simplification purposes, two cositing operators are considered as sufficient for the presented performance analysis.

The spectrum available for the auctioning game will be merged into a single pool of HSPA carriers, which will be available at each cell, and can be used by any operator in TDMA mode, depending on the auction outcomes in each cell. In case of non-shared carriers, frequency reuse 1 was considered.

The decision of which of the pooled and shared resources are to be used by each operator for particular time period $\Delta$ is taken by the AU and its decision will be valid for a time period of duration $\Delta$, after which the Auctioning Process (AP) must be repeated and new resources allocation scheme shall be provided. At each time interval, operators calculate their valuations for resources in the next interval. Valuation information is forwarded to the cell specific AU unit. The AU's decisions will be made based on submitted valuations.

A valuation is a function that encodes the amount of profit a particular operator expects from using a certain number of resources in the next time period. How to optimally calculate such function is not obvious and may differ from operator to operator. However, we show that already the simple valuations from Section III A lead to substantial spectrum utilization gains.

NOTE: Proposed auction-based spectrum sharing can be seen as a RAT independent solution, as far as regulators and the specification allow certain frequency band to be used by other RAT's. For the purpose of this analysis, single RAT was selected for performance evaluation. Inter-RAT evaluation is out of scope of this paper.

\section{SYSTEM LEVEL NETWORK MODEL}

System model consists of 19 3-sectorized, homogeneous cells in wrap around configuration. We assume, that two MNO operators who are willing to participate in the auction based spectrum sharing, are co-sited, providing coverage over the same geographical area and serve their own subscribers only (i.e. national roaming disabled). UE locations were generated randomly.

Assumed co-located BS' enforcement might be seen as a limitation from the network planning perspective, but on the 


\begin{tabular}{|c|c|}
\hline Parameter & Value and comment \\
\hline Network layout & Hexagonal grid, 19 sites/57 sectors \\
\hline Wrap around & Yes \\
\hline Network operators & Two operators, co-sited \\
\hline Spectrum auctions setup & Auctions running in all cells \\
\hline Inter site distance & $500 \mathrm{~m}$ \\
\hline Terminal distribution & Random UE locations in all cells \\
\hline Cell Isolation & $0 \mathrm{~dB}$ \\
\hline Carrier frequency & $2 \mathrm{GHz}$ \\
\hline RAT & MC-HSDPA \\
\hline Number of bands & 1 \\
\hline BS antenna configuration & 3 sector \\
\hline Antenna beamwidth & $70 \mathrm{deg}$ \\
\hline Antenna Front To Back ratio & $20 \mathrm{~dB}$ \\
\hline NodeB antenna gain & $14 \mathrm{dBi}$ \\
\hline NodeB Tx power & $43 \mathrm{dBm}$ \\
\hline Minimum UE to BS distance & $35 \mathrm{~m}$ \\
\hline Propagation model & $128.1+37.6 \log 10(\mathrm{R}) ; \mathrm{R}[\mathrm{km}]$ \\
\hline Shadow fading & $8 \mathrm{~dB}$ \\
\hline Shadowing correlation & 1 between sectors, 0.5 between sites \\
\hline Penetration loss & $0 \mathrm{~dB}$ - indoor scenario not considered \\
\hline Thermal noise level & $-102.9 \mathrm{dBm}$ \\
\hline Channel model & PedA $3 \mathrm{~km} / \mathrm{h}$ \\
\hline Fading across carriers & Uncorrelated \\
\hline Simulation duration & $10 \mathrm{sec}$. \\
\hline Traffic & Bursty traffic \\
\hline Scheduler & Proportional Fair \\
\hline
\end{tabular}

Table I

SELECTED SYSTEM LEVEL PARAMETERS

other hand, it opens the possibility to re-use the RF components from the other auction player's infrastructure, which might be a serious advantage in many cases. Moreover, there will be need to exchange certain amount of auctioning-related control information - BS co-location might ease practical realization of such information flow. Detailed solution, as well as standardization related analysis, is out of scope of this paper. In the Table I, more details on the system level model were provided.

\section{A. Spectrum resources consideration}

Spectrum resources allowed to be used by the AU in auctions were proposed to be pooled in one set, as it was described in Section IV. The most straight forward use-case, would be to have (at least) two collocated operators, who are willing to allow certain percentage of their spectrum (expressed in the number of HSPA carriers in this case) for auctioning purposes. It shall be kept in mind, that auctioned carriers (AUC) can be accessed by any of the auction participants, under the TDMA sharing principles. This kind of the spectrum sharing scheme is also called orthogonal spectrum sharing [15]. Furthermore, we assume that licensed bands are considered for described scenarios and all auction players have equal (or, proportional to the amount of shared radio resources) priorities in accessing AUC carriers. For sake of simplicity, we have assumed that each of the operators have deployed Multi Carrier HSDPA network and each of them agreed to assign number of owned HSPA carrier for the auctioning pool. As we assume that the available channels are of identical value, the decision to be made is the number of channels to be allocated to each of

\begin{tabular}{|c|c|}
\hline Parameter & Value \\
\hline \hline Number of packets per burst & 10 \\
\hline Packet arrival rate & 0.1 sec. \\
\hline Burst size & Variable \\
\hline Packet size within burst & Fixed \\
\hline UE's per cell & 10 \\
\hline
\end{tabular}

Table II

TRAFFIC MODEL PARAMETERS

the operators. NOTE: The radio resources to be pooled for the auctioning purposes might be also provided by third party player, e.g. broadband wireless provider.

\section{B. Traffic Model}

It is important to note that the resources sharing scenario is meaningful only in case of highly loaded networks, or in other words, in case of capacity limited networks. From practical point of view: no MNO is expected to ask for additional spectrum resources, in case when the currently owned spectrum resources are sufficient for smooth operation of particular network. For that reason, traffic model is seen as crucial aspect in the presented analysis due to the fact, that the used valuation function was simply modeled as the cell specific data buffer size for each of operators (i.e. bids for the multi-unit auctioning purposes were modeled by the variable cell load and proportional fairness). Therefore, it was expected, that the operator with highly loaded network is going to use the shared resources more frequent than the other operator.

Aspects of the equal traffic model among auction participants were investigated in [18], also considering various spectrum exchange algorithms in HSPA and LTE network. Orthogonally modeled traffic arrival was proposed in [16]. In this paper, we evaluate HSPA system performance by considering a more realistic, partially correlated load balance among two operators, introducing tolerance on top of the reference offered cell load in order to generate operator specific load. For that purpose, tolerance of \pm 10 and \pm 50 were selected to be used in the simulations. The smaller value was selected to simulate the sharing performance among two comparable operators. The second case was defined for less uniform market situation, e.g. MNO's focusing on certain subscriber's classes. Traffic model was based on the operator's specific demands, being defined based on the packet burst size. Number of the active subscribers using data services was fixed and constant for both operators in all sectors.

\section{Simulation Results}

In this section we present and analyze simulation results based on the system model as described above, focusing on the total goodput (application level throughput, not accounting the retransmissions and overheads) figures for both evaluated MNO's, i.e. not considering individual operator's gains and pains coming from the discussed solution, which were described in [18]. In the first step, we look at the throughput 50\%-tile comparison for three scenarios, where only one carrier was released by each auction player to be pooled for 


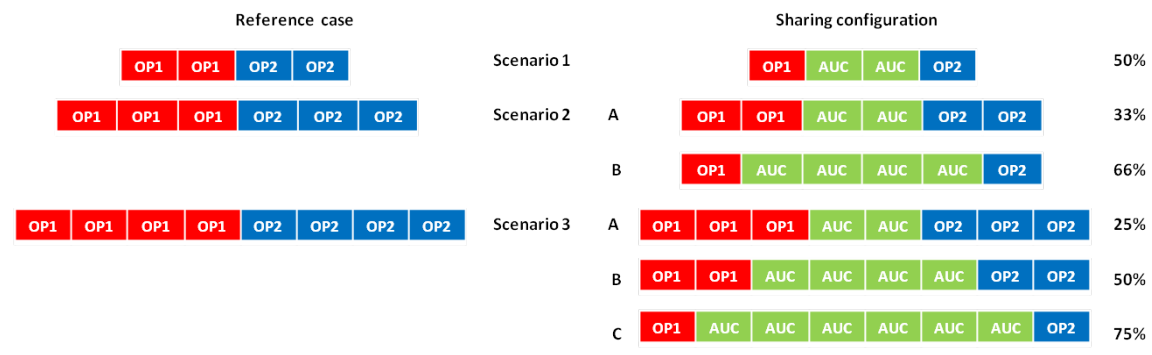

Figure 2. Carrier allocation scheme and shared carriers percentage; OP1: Operator1 only, OP2: Operator2 only, AUC: Auctioned carriers - Operator1 or Operator2

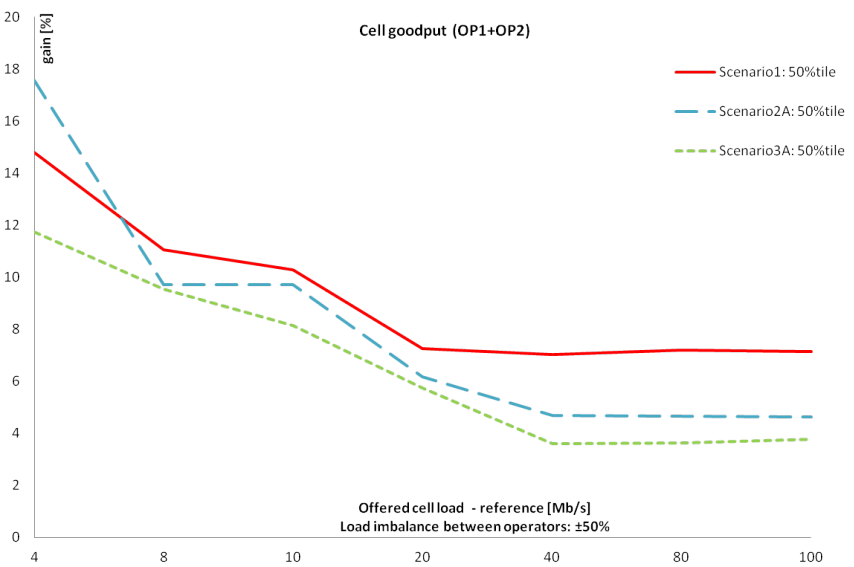

Figure 3. Single HSPA carrier sharing comparison, for various scenarios

sharing purposes (i.e. Scenario 1, 2A and 3A). As depicted on Figure 3, consideration of only one carrier per MNO being pooled for sharing purposes allowed generation of visible gains in terms of the total goodput, which can be easily translated into the increased spectrum utilization. Achieved sharing gain varies depending on the cell load and saturates for highly loaded network scenarios.

In the next step, we investigate the HSPA carrier's configuration impact on the sharing gain over the respective reference cases, as a function of the cell load. As depicted on Figure 2 , we have defined two scenarios, where $50 \%$ of the owned carriers were shared by both network operators: these are Scenario 1 and $3 \mathrm{~B}$ - these configurations were selected for comparison in order to keep fixed sharing ratio as parameter.

Based on the results presented on Figure 4, it was observed that despite of evident sharing gains achieved in both scenarios in terms of total spectrum utilization, in can be additionally concluded, that for particular sharing percentage (i.e. $50 \%$ in this case), the cooperation results for each of the depicted throughput percentiles are reaching the saturation levels for the increasing cell load values. Furthermore, the introduced saturation levels can be considered as constant and stable. Based on this observation, the next step in the results analysis was the evaluation of sharing percentage impact on the total cell goodput.

On Figure 5, we can observe, that in the evaluated scenarios,

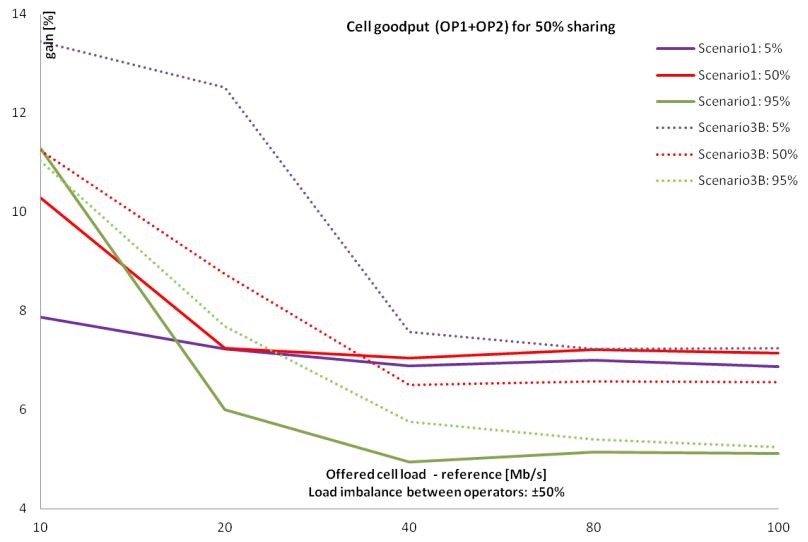

Figure 4. Total goodput for 50\% sharing scenarios: 5, 50 and $95 \%$ tile comparison

the higher spectrum resources sharing factor was, the higher gains were achieved in terms of total cell goodput. In general, this conclusion holds for all analyzed throughput percentiles. In most cases, the highest gains were observed for 5\%-tiles of the cell goodput's, what can be translated into the cell edge users improvements.

Based on this observation, it would be interesting to look at larger carrier's configurations, but due to the HSPA carriers spectrum requirements, higher spectrum configurations were seen as not too realistic. Therefore, further study will be continued based on the LTE network, what is out of scope of this work.

The curves depicted on Figure 6 ( \pm 50 load imbalance) have non-increasing trends, which might give an impression, that there might be even higher gains opportunities for cell load values lower that those presented on the enclosed figures. However, a limit to these gains comes from the fact that sharing brings benefits only in case of capacity limited scenarios. Once we have cell specific offered load low enough, we do not need to participate in the described auctioning game (the exclusively owned spectrum resources are sufficient to serve the offered load) - therefore the sharing gain is no longer visible. Moreover, comparing simulation results for various load imbalance ratios between operators (i.e. \pm 10 and \pm 50 , as depicted on Figure 5 and 6, respectively), one can notice, that the higher sharing gains were achieved in case of larger 


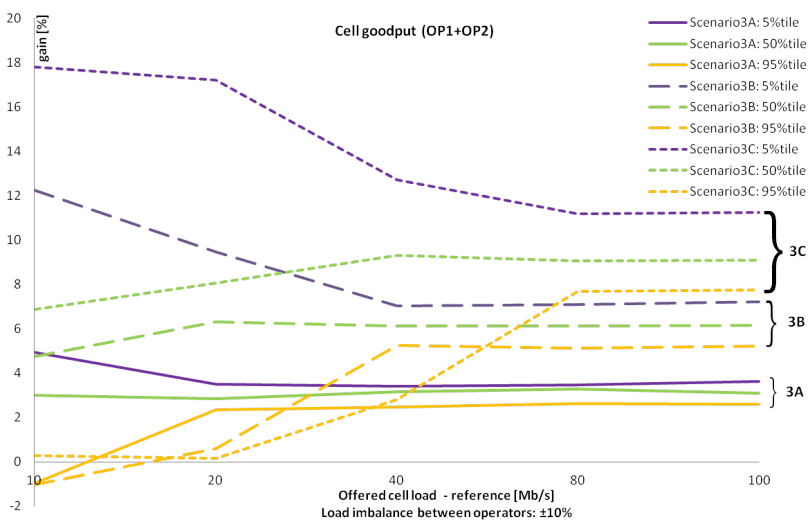

Figure 5. Total goodput for Scenario 3A, B, C for 5\%, 50\% and 95\%tile

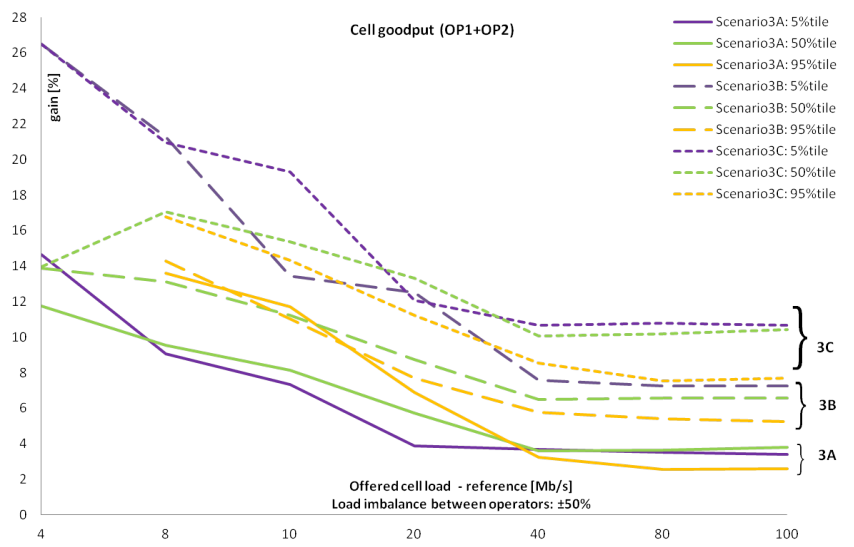

Figure 6. Total goodput for Scenario 3A, B, C for 5\%, 50\% and 95\%tile

load imbalance. This can be easily explained by the fact, of simple resources re-use by the network which has higher load, utilizing the spectrum resources of the operator with lower valuations provided during auctions. Once the load imbalance between two networks is decreasing, the valuation functions do not allow any of the auction players to become much stronger and dominant, but as presented above, sharing gains are still clearly visible across various radio conditions.

Based on the presented spectrum utilization gains, it was concluded, that the described spectrum sharing algorithm extended with appropriate business model for the operators coalition formation, might bring additional money revenue gains to the network operators.

\section{CONCLUDING REMARKS}

We have observed, that the short time interval spectrum sharing in the collocated HSDPA network, can provide the spectrum utilization gains, despite of low spectrum granularity of the HSPA physical layer. Gains were presented in case of low to medium granularity of auctioned spectrum resources. Furthermore, traffic patterns de-correlation proves to offer global spectrum utilization improvements. It is expected, that consideration of different valuation functions, as well as other scheduling rules will also impact the results, what is left for further study. The auction based resources distribution system as proposed, besides technically improving spectrum utilization, can also be seen as a tool to monetize the spectrum resources, or even to release chunks of unused licensed spectrum for opportunistic secondary usage.

The introduced valuation functions can easily be used to assign monetary values to the radio resources. The example of Google Adwords market (i.e. pay-per-usage) clearly demonstrates, that such monetization stimulates participation and may lead to the development of new services. The proposed solution may also be seen as a tool to create a transparent market of radio resources enhancing the revenue generation as well as creating new business models on the mobile services market.

ACKNOWLEDGMENT: The research leading to these results has received funding from the European Community's Seventh Framework Programme FP7/2007-2013 under grant agreement n\#248001 - SAPHYRE.

\section{REFERENCES}

[1] Kumar, S. Costa, G. Kant, S. Frederiksen, F.B. Marchetti, N. Mogensen, P. Spectrum sharing for next generation wireless communication networks, Cognitive Radio and Advanced Spectrum Management, 2008 I

[2] V. Heinonen, P. Pirinen, and J. Iinatti, Capacity gains through interoperator resource sharing in a cellular network, in Proc. IEEE WPMC, 2008 I

[3] Xinsheng Zhao Zhiyi Guo Qiang Guo, A cognitive based spectrum sharing scheme for LTE advanced systems, ICUMT, 2010 I

[4] Network Sharing in LTE - Opportunity \& Solutions, Resource Center/White Papers, Alcatel-Lucent, (2010) I

[5] Berkers, F. Hendrix, G. Chatzicharistou, I. de Haas, T. Hamera, D. To Share Or Not To Share? Business aspects of network sharing for Mobile Network Operators, Intelligence in Next Generation Networks (ICIN), 2010

[6] Frisanco, T., P. Tafertshofer, P. Lurin, and R. Ang, Infrastructure sharing and shared operations for mobile network operators: From a deployment and operations view, IEEE International Conference on Communications, pp. 2193-2200, 2008 I

[7] Mobile Infrastructure Sharing, GSMA, retrieved on March 7th, 2011 from http://www.gsmworld.com/documents/gsma.pdf IV

[8] N. Nisan, T. Roughgarden, E. Tardos, V.V. Vazirani. Algorithmic Game Theory, Cambridge University Press, 2008 II

[9] S. Khot, R.J. Lipton, E. Markakis, and A. Mehta. Inapproximability results for combinatorial auctions with submodular utility functions. In WINE 2005 (LNCS 3828), pages 92-101 II

[10] Shahar Dobzinski, Noam Nisan, and Michael Schapira. Truthful randomized mechanisms for combinatorial auctions. In STOC06 II

[11] Daniel Lehmann, Liadan Ita OCallaghan, and Yoav Shoham. Truth revelation in approxi- mately efficient combinatorial auctions. In JACM 49(5), pages 577602, Sept. 2002 II

[12] Vickrey, W. (1961) Counterspeculation, auctions, and competitive sealed tenders. Journal of Finance, 16, 8-37 II

[13] Clarke, E. (1971) Multipart pricing of public goods. Public Choice, 11, 1733 II

[14] Groves, T. (1973) Incentives in teams. Econometrica, 41, 617631 II

[15] FP7 SAPHYRE Sharing Physical Resources - Mechanisms and Implementations for Wireless Networks. project, www.saphyre.eu, 2011 V-A

[16] Litjens R., Zhang H. Analytical assessment of the performance impact of spectrum sharing in cellular networks considering different time scales EUSIPCO 2011, 176-180 V-B

[17] Jianwei Huang, Randall A. Berry, Michael L. Honig Auction-based Spectrum Sharing WiOpt'04 2004 I

[18] Torsten Fahldieck, Michał Szydełko, Haibin Zhang, Martin Schubert, Luca Anchora, Jianshu Zhang, Resource allocation and interference management strategies, SAPHYRE deliverable D4.1, 2012

$\mathrm{V}-\mathrm{B}, \mathrm{VI}$ 